\title{
Exploring The Mechanism of Action of Herbal Medicine (Gan-Mai-Da-Zao Decoction) For Post- Stroke Depression Based On Network Pharmacology And Molecular Docking
}

\author{
Zhi-Cong Ding \\ Jinan University \\ Fang-Fang $\mathrm{Xu}$ \\ Jinan University \\ Qi-Di Sun \\ Yangzhou University \\ Bin Li \\ Jinan University \\ Neng-Xing Liang \\ Jinan University \\ Jun-Wei Chen \\ Jinan University \\ Shang-Zhen Yu ( $\nabla$ ysz6521@163.com ) \\ Jinan University https://orcid.org/0000-0003-0930-6092
}

\section{Research}

Keywords: post-stroke depression, Gan-Mai-Da-Zao decoction, network pharmacology, GO and KEGG pathway enrichment, molecular docking

Posted Date: May 17th, 2021

DOI: https://doi.org/10.21203/rs.3.rs-508953/v1

License: (c) (1) This work is licensed under a Creative Commons Attribution 4.0 International License. Read Full License 


\section{Abstract}

Backgrounds: Post-stroke depression is the most common and serious neuropsychiatric complication occurring after cerebrovascular accidents, seriously endangering human health while also imposing a heavy burden on society. Even so, it is difficult to have drugs to contain the progression of the disease. It's reported that Gan-Mai-Da-Zao decoction was effective to PSD, but it is unknown on its mechanism of action for PSD. In this study, we aimed to explore the possible mechanisms of action of Gan-Mai-Da-Zao decoction in the treatment of PSD using network pharmacology and molecular docking.

Material and methods: We obtained the active components and their targets of all drugs from the public database TCMSP and published articles. Then, we collected the PSD-related targets from GeneCards and OMIM databases. Cytoscape 3.8.2 was applied to construct PPI and composite target disease networks. In parallel, the DAVID database was used to perform GO and KEGG enrichment analysis to obtain the biological processes involved in drug treatment diseases in vivo. Finally, molecular docking was used to verify the association between the main active ingredients and the targets.

Results: The network pharmacological analysis of Gan-Mai-Da-Zao decoction for PSD identified 107 active ingredients with important biological effects, including quercetin, luteolin, kaempferol, naringenin, isorhamnetin, etc. A total of 203 potential targets for drug treatment of diseases were screened, including STAT3, JUN, TNF, TPT53, AKT1, EGFR, etc. They were found to be widely enriched in a series of signaling pathways such as TNF, HIF-1, and the Toll-Like receptor. Meanwhile, molecular docking analysis showed that the core active components were tightly bound to the core targets, further confirming their anti-PSD effects.

Conclusion: This is a prospective study based on the integration and analysis of large data, using the technology of network pharmacology to explore the feasibility of Gan-Mai-Da-Zao decoction for the treatment of PSD, and successfully validated by molecular docking. It reflects the multi-component and multi-target characteristics of Chinese medicine, and more importantly, it also brings hope to the clinical treatment of PSD.

\section{Introduction}

Post-stroke depression (PSD) is one of the most common and heavy neuropsychiatric complications after stroke [1, 2], which often starts insidiously, with mild symptoms of malaise and drowsiness in the early stages. What's more, if patients with PSD are unable to express their feelings clearly due to language or cognitive impairment, the diagnosis is often compromised and treatment is delayed [3]. This not only poses a great challenge to clinical work but also adds a heavy burden to the society and economy. A recent statistical study showed that approximately 795,000 people suffered from stroke each year in the United States. In detail, approximately 610,000 people of them had a stroke for the first time, and about 185,000 people have recurred stroke, more than 100,000 of those people die from stroke [4]. A meta-analysis of longitudinal studies found that the prevalence of depression was $29 \%(95 \% \mathrm{Cl} 25-32)$, 
and remains stable up to 10 years after stroke, with a cumulative incidence of $39-52 \%$ within 5 years of stroke [5]. Studies reported the cross-sectional prevalence of PSD was $18 \%$ and $33 \%$ [5-8]. So far, tricyclic antidepressants (TCAs) and selective serotonin reuptake inhibitors (SSRIs) were used to treat PSD.

However, due to the lack of timely diagnosis, the adverse effects on cardiovascular function of drugs, and increased risk of bleeding, which leads to unsatisfactory treatment of patients with $\operatorname{PSD}[9,10]$.

Traditional Chinese medicine (TCM) for post-stroke depression is characterized by fewer side effects and individualized treatment [11]. Therefore, the search for the treatment of PSD from herbal medicine has become a hot topic in contemporary pharmacological research. Gan-Mai-Da-Zao decoction is from Jingui Yaolue, which is composed of three Chinese herbal medicines: Glycyrrhiza uralensis Fisch. (gancao in Chinese), Triticum aestivum L. (xiaomai in Chinese), and Ziziphus jujuba Mill. (dazao in Chinese), which is mainly used for sleep disorders and depression-related psychiatric disorders [12]. It's reported Gan-MaiDa-Zao decoction intervention 2 and 4 weeks could significantly improve Hamilton depression rating scale (HAMD) scores in postpartum women with depressive states in a randomized controlled study [13]. Modern pharmacological studies have also confirmed Gan-Mai-Da-Zao decoction could increase central excitability, sedation and hypnotism, anti-depressant and other pharmacological activities [14-16]. However, it's unclear about the mechanism of the active ingredients in Gan-Mai-Da-Zao decoction to alleviate depressive symptoms.

TCM with multiple components, multiple targets, and multiple pathways can bring very many new possibilities for clinical treatment. Network pharmacology is the result of the integration of multidisciplinary basic theories and research tools such as biology, computer science, multidirectional pharmacology, molecular pharmacology, and medicine, which can systematically and comprehensively reflect the intervention mechanism of drugs on disease networks. This has a strong convergence with the principle of overall dynamics of TCM treatment of diseases and the characteristics of multi-component, multi-target, and multi-pathway interactions. Therefore, network pharmacology can provide a new and powerful technical support for the study of the mechanism of action of TCM compounding, which can help to reveal the scientific connotation of TCM compounding, discover drug targets, inherit and develop TCM theory [17]. This experiment aims to investigate the mechanism of action of Gan-Mai-Da-Zao decoction in the treatment of PSD through network pharmacology and molecular docking technology (Fig. 1).

\section{Materials And Methods}

\section{Screening of active compounds and prediction of putative targets}

Through the traditional Chinese medicine systems pharmacology database and analysis platform (TCMSP, https://tcmspw.com/tcmsp.php), an authoritative public database that collects a large number of active ingredients, related targets, and pharmacokinetic information [18], we searched for the active ingredients of three Chinese medicines and supplemented them with published literature [19, 20]. Oral 
bioavailability $(\mathrm{OB})$ is an important indicator for evaluating the rate and extent of drug absorption into the human circulation, and drug-likeness (DL) is an indicator for evaluating the similarity of a compound to a known drug [21, 22]. Based on these two ADME (absorption, distribution, metabolism, and excretion) mode values, we performed a preliminary screening of active ingredients to obtain the active compounds and their protein targets, where $\mathrm{OB} \geq 30 \%$ and $\mathrm{DL} \geq 0.18 \%$ were set to obtain the above information [23]. After screening, to standardize protein target information, the Uniprot database (The Universal Protein Resource, https://www.uniprot.org/) was unified to standardize the protein targets on which compounds act, resulting in a piece of more comprehensive target information such as gene IDs and gene symbols.

\section{Identification of disease-related targets and filtering intersecting targets}

Using "Post-Stroke Depression" as the keyword, we mined the GeneCards database (https://www.genecards.org/) and the OMIM database (https://omim.org/) for gene targets related to PSD [24]. The targets associated with PSD were obtained by merging the targets of the two databases and removing duplicates. The common targets were then screened by using Venny 2.1 (http://bioinformatics.psb.ugent.be/webtools/Venn/), and the common targets were defined as the potential targets of Gan-Mai-Da-Zao decoction for PSD.

\section{Protein-protein interaction construction}

A Protein-protein interaction (PPI) network model was constructed by submitting common disease-drug targets to the search tool for the retrieval of interacting genes (STRING, https://string-db.org/) database [25]. Set the organism species as "Homo sapiens", and the confidence score with correlation degree $\geq$ 0.950 , meanwhile hide disconnected nodes. Then, the interaction information was further visually analyzed by Cytoscape 3.8.2. The CytoNCA plug-in can analyze the topological attributes of the data submitted to Cytoscape [26, 27]. In this plug-in, betweenness centrality (BC), closeness centrality (CC), and degree centrality (DC) are used to estimate the importance of nodes in the network. The higher the quantitative value of these three numerical values, the more important the node is in the network. In the PPI network, the values of $\mathrm{BC}, \mathrm{CC}$, and DC are used as variables to screen out the core targets and build a network relationship diagram of the core targets based on the screening results.

\section{GO and KEGG pathway enrichment analysis}

The previously collected Gan-Mai-Da-Zao decoction targets for the treatment of PSD were imported into the DAVID (https://david.ncifcrf.gov/summary.jsp) database to analyze its main biological processes and metabolic pathways and enrich them set of analysis, which is a comprehensive annotation capability and have updated monthly gene annotation data repository. In species selection, we chose "Homo sapiens", terms with a $P<0.05$. The data results were saved and visualized using bioinformatics (http://www.bioinformatics.com.cn/) to finally obtain The Gene Ontology (GO) and Kyoto Encyclopedia of Genes and Genomes (KEGG) enrichment analysis bubble map, where $G 0$ enrichment analysis covers 
three aspects of biology: cellular component, molecular function, biological process, KEGG enrichment analysis can also suggest us the biological mechanism of drug action in the human body and the pathways involved in regulation.

\section{Network construction}

(1) Cytoscape 3.8.2 was implemented to draw out the disease-herb-component-target (D-H-C-T) network relationship diagram to visualize the relationship between Gan-Mai-Da-Zao decoction and PSD. The different colors and shapes in the diagram represent the disease, drug, component, and target, and the "edges" represent the correlation between this information. (2) To more visually reflect the relationship of target enrichment on the pathway, a more complex but more intuitive network relationship diagram was constructed by combining the previous network relationship diagram, which includes the more comprehensive information of the drug, active ingredient, target, and pathway.

\section{Molecular docking prediction}

\section{Preparation of small molecule ligands}

To better evaluate the reliability of network analysis predictions, the core active ingredient was then molecularly docked to the core gene target. Firstly, the small molecule ligands were prepared by obtaining the 2D structures of the active ingredients in sdf format through PubChem

(https://pubchem.ncbi.nlm.nih.gov/) database and then converting the 2D structures to 3D structures in mol2 format through ChemOffice software. Finally, it was imported into AutoDock-Tools to convert to pdbqt format files [28].

\section{Preparation of protein receptors}

Firstly, the core gene target was entered into the Uniprot database to obtain its Uniprot ID, and the 3D structure of the core gene target was retrieved and downloaded from Protein Data Bank (PDB, http://www.rcsb.org/) database in pdb format by Uniprot ID. Using PyMOL 2.3.4 software, the protein was de-watered and de-liganded, and the core gene target was hydrogenated and charge calculated using AutoDock-Tools [29], and finally saved as a pdbqt format file. When everything was ready, ligand-receptor molecular docking was performed using AutoDock Vina.

\section{Results}

\section{Active compounds and targets of gancao, xiaomai and dazao}

We found a total of 442 drug-related components from the TCMSP database as well as from the available literature. Then the screening was performed according to $D L \geq 0.18$ and $O B \geq 30 \%$, meanwhile, the gene targets involved in each active ingredient were obtained, and finally, the duplicate targets were removed after comparison and correction by the Uniprot database. A complete of 109 active ingredients 
and 239 gene targets were obtained, among which some active ingredients and targets were derived from multiple drugs, which also reflects the multi-component and multi-target characteristics of TCM. The details about active ingredients are shown in the Supplementary file, Table S1.

\section{Gene targets of PSD}

In this study, 4733 PSD-related gene targets were obtained from the GeneCards database, and another 37 gene targets were obtained from the OMIM database, in which there were 8 identical targets, after removing duplicate values, the total number of PSD-related gene targets was 4762. Next, 203 interacting gene targets were obtained by taking the intersection of disease-related gene targets and drug-related gene targets (Fig. 2a). These targets are the potential targets of the Gan-Mai-Da-Zao decoction against PSD (Table 1).

\section{PPI network analysis}

The 203 intersecting gene targets were submitted to the STRING database, and then the data obtained from this platform were imported into Cytoscape 3.8.2 for visualization and topological analysis (Fig. 2b), which consisted of 139 nodes and 426 edges, and the topological properties of the intersecting gene targets were analyzed using the CytoNCA plug-in, whose median values of BC, CC, and DC were: 22, 0.046184739 , and 4 . There were 49 gene targets above the median, and these genes were important for the treatment of PSD with Gan-Mai-Da-Zao decoction (Fig. 2c). Among them, AKT1, STAT3, TP53, CTNNB1, CDKN1A, ESR1, VEGFA, MAPK1, MAPK3, CASP8, CCND1, MAPK14, RELA, TNF, EGFR, FOS, JUN, CXCL8, STAT1 were greater than the twofold median values of BC, CC, DC $(286.3560165,0.046954747$, 11), indicating that these 19 genes are the core targets in the PPI network (Fig. 2d). The details from STRING are shown in the Supplementary file, Table S2.

Table 1 Potential targets of Gan-Mai-Da-Zao decoction against Post-Stroke Depression (PSD) 


\begin{tabular}{|c|c|c|c|c|c|c|c|c|c|}
\hline NO. & Gene & No. & Gene & NO. & Gene & No. & Gene & NO. & Gene \\
\hline 1 & PTGS1 & 42 & PLAU & 83 & CDKN1A & 124 & THBD & 165 & CCNA2 \\
\hline 2 & DRD1 & 43 & LTA4H & 84 & MMP9 & 125 & SERPINE1 & 166 & ESR2 \\
\hline 3 & CHRM1 & 44 & MAOA & 85 & MAPK1 & 126 & COL1A1 & 167 & CDK2 \\
\hline 4 & DRD5 & 45 & ADRB1 & 86 & IL10RA & 127 & IFNG & 168 & MAPK10 \\
\hline 5 & SCN5A & 46 & BCL2 & 87 & EGF & 128 & ALOX5 & 169 & PYGM \\
\hline 6 & CHRM5 & 47 & BAX & 88 & RB1 & 129 & IL1A & 170 & GRIA2 \\
\hline 7 & PTGS2 & 48 & CASP9 & 89 & TNF & 130 & MPO & 171 & OLR1 \\
\hline 8 & HTR3A & 49 & JUN & 90 & IL6ST & 131 & NCF1 & 172 & IL4 \\
\hline 9 & RXRA & 50 & CASP3 & 91 & AHSA1 & 132 & ABCG2 & 173 & HSD3B1 \\
\hline 10 & OPRD1 & 51 & CASP8 & 92 & TP53 & 133 & GSTP1 & 174 & IKBKB \\
\hline 11 & SLC6A2 & 52 & PRKCA & 93 & ELK1 & 134 & NFE2L2 & 175 & MAPK8 \\
\hline 12 & ADRA1A & 53 & PON1 & 94 & NFKBIA & 135 & NQ01 & 176 & PPP3CA \\
\hline 13 & CHRM2 & 54 & MAP2 & 95 & POR & 136 & PARP1 & 177 & AKR1C3 \\
\hline 14 & ADRA2B & 55 & CAT & 96 & ODC1 & 137 & AHR & 178 & SLPI \\
\hline 15 & ADRA1B & 56 & HAS2 & 97 & TOP1 & 138 & SLC2A4 & 179 & MAPK3 \\
\hline 16 & SLC6A3 & 57 & DRD4 & 98 & RAF1 & 139 & COL3A1 & 180 & LDLR \\
\hline 17 & ADRB2 & 58 & $\mathrm{ACHE}$ & 99 & SOD1 & 140 & CXCL11 & 181 & BAD \\
\hline 18 & CHRNA2 & 59 & F7 & 100 & HIF1A & 141 & CXCL2 & 182 & MTTP \\
\hline 19 & SLC6A4 & 60 & CACNA1S & 101 & STAT1 & 142 & NR1I3 & 183 & APOB \\
\hline 20 & DRD2 & 61 & KDR & 102 & RUNX1T1 & 143 & CHEK2 & 184 & PLB1 \\
\hline 21 & OPRM1 & 62 & AKT1 & 103 & HSPA5 & 144 & CLDN4 & 185 & HMGCR \\
\hline 22 & GABRA1 & 63 & VEGFA & 104 & ERBB2 & 145 & PPARA & 186 & CYP19A1 \\
\hline 23 & NR3C2 & 64 & MMP2 & 105 & ACACA & 146 & PPARD & 187 & UGT1A1 \\
\hline 24 & PPARG & 65 & MMP1 & 106 & CYP1A1 & 147 & HSF1 & 188 & SREBF1 \\
\hline 25 & CYP3A4 & 66 & HMOX1 & 107 & ICAM1 & 148 & CXCL10 & 189 & GSR \\
\hline 26 & NR1/2 & 67 & CYP1A2 & 108 & IL1B & 149 & CHUK & 190 & $\mathrm{ABCC} 1$ \\
\hline 27 & CYP2B6 & 68 & CAV1 & 109 & CCL2 & 150 & SPP1 & 191 & ADIPOR1 \\
\hline 28 & NOS2 & 69 & CTNNB1 & 110 & SELE & 151 & RUNX2 & 192 & ABAT \\
\hline
\end{tabular}




\begin{tabular}{|llllllllll|}
29 & KCNH2 & 70 & MYC & 111 & VCAM1 & 152 & E2F1 & 193 & SOAT1 \\
\hline 30 & ESR1 & 71 & CASP7 & 112 & PTGER3 & 153 & CTSD & 194 & BACE2 \\
\hline 31 & AR & 72 & F3 & 113 & CXCL8 & 154 & IGFBP3 & 195 & STAT3 \\
\hline 32 & PRSS1 & 73 & GJA1 & 114 & PRKCB & 155 & IGF2 & 196 & CDK4 \\
\hline 33 & PDE10A & 74 & MMP10 & 115 & BIRC5 & 156 & CD40LG & 197 & MDM2 \\
\hline 34 & MAOB & 75 & FASN & 116 & DUOX2 & 157 & IRF1 & 198 & APP \\
\hline 35 & ADRA2A & 76 & DPP4 & 117 & NOS3 & 158 & ERBB3 & 199 & PCNA \\
\hline 36 & CA2 & 77 & MMP3 & 118 & HSPB1 & 159 & HK2 & 200 & TYR \\
\hline 37 & ADRA2C & 78 & RELA & 119 & SULT1E1 & 160 & RASA1 & 201 & XIAP \\
\hline 38 & ADRA1D & 79 & EGFR & 120 & IL2RA & 161 & GSTM1 & 202 & PTGES \\
\hline 39 & PGR & 80 & CCND1 & 121 & CYP1B1 & 162 & MAPK14 & 203 & MET \\
\hline 40 & ADH1C & 81 & BCL2L1 & 122 & CCNB1 & 163 & GSK3B & & \\
\hline 41 & AKR1B1 & 82 & FOS & 123 & PLAT & 164 & CHEK1 & & \\
\hline
\end{tabular}

\section{GO and KEGG Pathway Enrichment Analyses}

The information retrieved from the DAVID database was ranked into TOP20 according to the $p$-value, and finally, the data was transformed into a bubble chart for display. When more genes were enriched the larger the bubble was and the deeper the color the smaller the $p$-value.

The biological process mainly involves response to drug (G0:0042493), response to lipopolysaccharide (G0:0032496), positive regulation of transcription from RNA polymerase II promoter (G0:0045944), response to ethanol (G0:0045471) and response to estradiol (G0:0032355) (Fig. 3a), and analysis on cellular components showed that it was similar to extracellular space (G0:0005615), cytosol (G0:0005829), membrane raft (GO:0045121), plasma membrane (G0:0005886) and integral component of plasma membrane (G0:0005887) are strongly related (Fig. 3b). Besides, molecular functions aspects mainly involve enzyme binding (G0:0019899), identical protein binding (G0:0042802), protein heterodimerization activity (G0:0046982), drug binding (GO:0008144), and protein binding (G0:0005515) (Fig. 3c). All of the above data suggest that the Gan-Mai-Da-Zao decoction may treat PSD by modulating multiple GO functions.

The KEGG pathway enrichment analysis yielded a total of 122 pathway information, and the same selection rules of GO enrichment analysis were used to obtain the TOP20 pathways for graphic visualization, through the pictures we can visually see that the functions of relevant targets for PSD were mainly enriched in Hepatitis B (hsa05161), 
Pathways in cancer (hsa05200), Pancreatic cancer (hsa05212), Bladder cancer (hsa05219) and TNF signaling pathway (hsa04668). Among these pathways, "Pathways in cancer" was identified as an important critical pathway with the highest target enrichment, and similarly, "Hepatitis B" was also an important pathway because it had the lowest $p$-value (Fig. $3 \mathrm{~d}$ ). The details about GO and KEGG enrichment analysis, which from the DAVID database are shown in the Supplementary file, Table S3.

\section{Network construction analysis}

There were 314 nodes and 1825 edges in the D-H-C-T network diagram, among which the potential active ingredients icos-5-enoic acid (MOL004985) and gadelaidic acid (MOL004996) were hidden in the network diagram because the corresponding gene targets didn't overlap with the disease targets (Fig. 4). The details are shown in the Supplementary file, Table S4. The pathway information was imported into Cytoscape 3.8.2 and combined with the previous graph to obtain a new network relationship graph with 335 nodes and 2300 edges (Fig. 5).

\section{Docking results}

The nodes with high degree values in PPI analysis were considered as core targets, and the five core gene targets with the highest degree values in PPI, STAT3 (PDB ID: 6NJS), JUN (PDB ID: 5T01), TP53 (PDB ID: 6WQX), AKT1 (PDB ID: 5WBL) and TNF (PDB ID: 2E7A) were correlated with the 10 active ingredients with the highest degree in the "D-H-C-T" network: quercetin (MOL000098), luteolin (MOL000006), kaempferol (MOL000422), 7-Methoxy-2-methyl isoflavone (MOL003896), naringenin (MOL004328), isorhamnetin (MOL000354), formononetin (MOL000392), licochalcone a (MOL000497), beta-sitosterol (MOL000358), and medicarpin (MOL002565) were molecularly docked and their binding energies were calculated (Table 2, Fig. 6). Meanwhile, we graphically demonstrated the specific details of the docking of the most core genes STAT3 and JUN with the most core components quercetin, luteolin, kaempferol, and 7Methoxy-2-methyl isoflavone (Fig. 7). The lower the binding energy of both ligand and receptor, the more stable the binding is [30]. In general, a docking fraction value of less than $-4.25 \mathrm{kcal} \cdot \mathrm{mol}^{-1}$ indicates some binding activity, less than $-5.0 \mathrm{kcal} \cdot \mathrm{mol}^{-1}$ indicates good binding activity, and less than -7.0 $\mathrm{kcal} \cdot \mathrm{mol}^{-1}$ indicates strong binding activity [31]. The results showed that a total of 50 groups of core components were selected to have good binding activity with the target proteins.

Table 2 Binding energies of 10 main compounds to two potential targets 


\begin{tabular}{|lllcccc|}
\hline NO. & Compound & \multicolumn{5}{c|}{ Binding Energy / $\mathrm{kcal}^{\prime} \mathrm{mol}^{-1}$} \\
\hline & & STAT3 & JUN & TP53 & AKT1 & TNF \\
\hline 1 & naringenin & -7.7 & -8.5 & -8.5 & -8.4 & -8.6 \\
\hline 3 & luteolin & -7.8 & -8.6 & -8.2 & -8.7 & -8.9 \\
\hline 4 & quercetin & -7.5 & -8.4 & -8.3 & -8.9 & -6.8 \\
\hline 5 & Medicarpin & -6.3 & -7.0 & -8.1 & -7.0 & -6.0 \\
\hline 6 & 7-Methoxy-2-methyl isoflavone & -7.6 & -8.6 & -9.0 & -8.4 & -6.7 \\
\hline 7 & beta-sitosterol & -7.2 & -6.6 & -7.0 & -7.3 & -6.2 \\
\hline 8 & formononetin & -7.4 & -7.8 & -8.6 & -8.3 & -8.4 \\
\hline 9 & isorhamnetin & -7.3 & -7.8 & -7.7 & -8.7 & -9.0 \\
\hline 10 & kaempferol & -7.3 & -7.8 & -7.9 & -8.4 & -8.9 \\
\hline
\end{tabular}

\section{Discussion}

Network pharmacology is a method to predict the possibility of drug treatment for diseases by searching for drug-disease shared genes and finding gene enrichment pathways, which are then confirmed by available experimental evidence. And molecular docking can predict the binding power of active ingredients to target proteins, which further confirms the therapeutic effect of drugs. The use of these techniques has largely solved the great challenges posed to research due to the multi-component and multi-target nature of TCM. Therefore, we used network pharmacology and molecular docking to reveal the possible mechanism of action of Gan-Mai-Da-Zao decoction against PSD.

In our study, the core active ingredients were screened in the "D-H-C-T" network, including quercetin, luteolin, kaempferol, naringenin, isorhamnetin, etc. In recent years, it has been found that flavonoids have significant effects on the central system, with neuroprotective, antidepressant, and anxiolytic effects [32]. In our study, quercetin is derived from Glycyrrhiza uralensis Fisch. and Ziziphus jujuba Mill. A relevant animal study confirmed that quercetin could reverse the stress-induced depression and anxiety in the mice [33]. Besides, it's evidenced that quercetin exerts anti-depressant effects through anti-oxidant, antiinflammatory, decreasing cytotoxicity, and increasing 5-hydroxytryptamine levels [34]. Luteolin is derived from Triticum aestivum $L$. It's reported that luteolin showed an anti-depressant effect via suppressing the endoplasmic reticulum [35], inhibiting and downregulating plasma membrane monoamine transporter (PMAT, Slc29a4) [36]. The study also found that luteolin may improve cognitive performance by inhibiting microglial activation and neuroinflammation in older mice [37]. Kaempferol, naringenin, and isorhamnetin are all derived from Glycyrrhiza uralensis Fisch. Kaempferol promoted the expression of brain-derived neurotrophic factor (BDNF) and nerve growth factor (NGF) protein in hippocampal tissue of aged rats in a 
chronic stress depression model, which resulted in neuroprotection and improved depression-like behavior [38]. Naringenin is a flavonoid compound with strong anti-oxidant and anti-inflammatory effects. The literature evidenced that naringin may produce functional behavioral effects through enhancement of cholinergic transmission, antioxidant defense systems, inhibition of lipid peroxidation and nitrosative processes [39]. The application of isorhamnetin potentiated the nerve growth factor (NGF) induced neurite outgrowth. In parallel, the expression of neurofilaments was markedly increased in the cotreatment of NGF and isorhamnetin in the cultures. This suggested that isorhamnetin might be used to some extent in the treatment of neurodegenerative diseases, including Alzheimer's disease and depression [40]. These important active ingredients are all sourced from Gan-Mai-Da-Zao decoction, and multiple active ingredients work together to exert their effects in the treatment of PSD.

In addition, a total of 19 core targets of Gan-Mai-Da-Zao decoction for PSD were screened in the PPI network, including AKT1, STAT3, TP53, CTNNB1, CDKN1A, ESR1, VEGFA, MAPK1, MAPK3, CASP8, CCND1, MAPK14, RELA, TNF, EGFR, FOS, JUN, CXCL8, STAT1. Among them, EGFR is highly expressed in a variety of malignancies, and depression is common in oncology patients, being four times more prevalent than in the general population [41, 42]. Meanwhile, it's also found that EGFR mutant non-small cell lung cancer produced depression by mediating inflammatory factors [43]. Depressant-like behavior was induced by forced swimming, and MAPK1 was overexpressed in the hippocampus to lead anti-depressant effect [44]. It's reported that estrogen-regulated neurotransmitter conversion and thus produced antidepressant effects. It's thought that the biological function of estrogen is largely mediated by intracellular activation of its primary receptors, estrogen receptor alpha (ESR1) and estrogen receptor beta (ESR2). Thus, genetic variation in ESR played an important role in the susceptibility of women to depression [45, 46]. STAT3 is expressed in both hippocampal neurons and glial cells and is closely related to neurodegenerative diseases. It was demonstrated that pharmacological treatment of PSD and improvement of depressive state may be related to the inhibition of JAK2/STAT3 signaling pathwayrelated gene and protein expression to promote the neural remodeling in the hippocampal [47].

Among the 20 pathways screened by KEGG enrichment analysis, some of them are closely related to PSD, including TNF and Toll-like receptor signaling pathways. The immune-inflammatory response is one of the important pathogenic mechanisms of PSD. Elevation of various inflammatory biomarkers such as IL6, TNF-a, increased high sensitive C-reactive protein (CRP) concentration was found to be present in mild to moderate depressive patients six months following stroke $[48,49]$. It has been shown that the improvement in depression in rats under acupuncture intervention may be closely related to the Toll-like receptor pathway and TNF signaling pathway enriched by significantly downregulated differentially expressed genes in the hippocampus, frontal lobes, and pituitary gland of rats [50]. Also, there are pathological mechanisms such as neuronal apoptosis and nerve growth disorders involved in some pathways that also play an important role in the development of PSD. The efficacy of current antidepressants has been linked to the Ras signaling pathway, which may be involved in the onset and development of depression-related disorders by indirectly affecting neurotrophic factors or directly affecting neuroplasticity [51]. Furthermore, antidepressants not only upregulate cAMP levels in receptor cells but also activate protein kinase $\mathrm{A}$ (PKA) to phosphorylate PKA, which then activates the cAMP- 
response element-binding protein (CREB) signaling pathway, altering functional protein activity and gene expression patterns to form new synapses, thus exerting antidepressant effects [52].

\section{Conclusion}

In summary, network pharmacological analysis showed that there are as many as 203 possible targets for Gan-Mai-Da-Zao decoction in the treatment of PSD. Several pathways may be very closely related to the treatment of PSD, including TNF and Toll-like receptor signaling pathways, and the 19 core gene targets screened from the PPI network map are also enriched in these important pathways. Therefore, the results of this study provide evidence for follow-up research and a basis for the clinical application of Gan-Mai-Da-Zao decoction and its prescriptions in the treatment of PSD.

\section{Abbreviations}

PSD: post-stroke depression

TCAs: tricyclic antidepressants

SSRIs: selective serotonin reuptake inhibitors

TCM: Traditional Chinese Medicine

HAMD: Hamilton Depression Rating scale

TCMSP: Traditional Chinese Medicine Systems Pharmacology Database and Analysis Platform

OB: Oral bioavailability

DL: Drug-likeness

ADME: absorption, distribution, metabolism, and excretion

Uniport database: The Universal Protein Resource

PPI: Protein-Protein Interaction

STRING: Search Tool for the Retrieval of Interacting Genes

BC: Betweenness Centrality

CC: Closeness Centrality

DC: Degree Centrality

GO: Gene ontology 
KEGG: Kyoto Encyclopedia of Genes and Genomes

D-H-C-T: disease-herb-component-target

PDB: Protein Data Bank

\section{Declarations}

\section{Acknowledgements}

The authors would like to thank the reviewers and also the authors of all references. The reviewer's advice really makes the great improvement of this paper.

\section{Authors' contributions}

DZC, XFF, and SQD: study design; acquisition of data; analysis of data. DZC and XFF wrote the manuscript. CJH, LB, and LNX: acquisition of data; critical revision of the manuscript. YSZ: revision of the manuscript and study supervision. All the author(s) read and approved the final manuscript.

\section{Availability of data and materials}

All data are available in the manuscript and they are exhibited in figures and tables.

\section{Ethics approval and consent to participate}

Not applicable.

\section{Consent for publication}

Not applicable.

\section{Funding}

This work is financially supported by the Research Fund of Traditional Chinese Medicine Bureau of Guangdong Province (No. 20183016), Science and Technology Projects in the field of health care in Jiangmen City, Guangdong Province (2021YL03021).

\section{Competing interests}

The authors declare that there are no conflicts of interest in relation to this work.

\section{References}

1. Shi Y, Yang D, Zeng Y, et al: Risk Factors for Post-stroke Depression: A Meta-analysis.Front Aging Neurosci. 2017;9: 
2. Martin TR, Oyiza M, Luis A, et al: Prevalence of pre-stroke depression and its association with poststroke depression: a systematic review and meta-analysis.Psychological Medicine. 2018;2018:1-12.

3. Zhang T: Stroke rehabilitation treatment guidelines in China.Chinese Journal of the Frontiers of Medical Science(Electronic Version). 2012;4:55-76.

4. Benjamin EJ, Muntner P, Alonso A, et al: Heart Disease and Stroke Statistics-2019 Update: A Report From the American Heart Association. 2019;139:e56-e528.

5. Ayerbe L, Ayis S, Wolfe CD, et al: Natural history, predictors and outcomes of depression after stroke: systematic review and meta-analysis.Br J Psychiatry. 2013;202:14-21.

6. Hackett ML, Pickles K: Part l: frequency of depression after stroke: an updated systematic review and meta-analysis of observational studies.Int J Stroke. 2014;9:1017-1025.

7. Jørgensen TS, Wium-Andersen IK, Wium-Andersen MK, et al: Incidence of Depression After Stroke, and Associated Risk Factors and Mortality Outcomes, in a Large Cohort of Danish Patients.JAMA Psychiatry. 2016;73:1032-1040.

8. Mitchell AJ, Sheth B, Gill J, et al: Prevalence and predictors of post-stroke mood disorders: A metaanalysis and meta-regression of depression, anxiety and adjustment disorder.Gen Hosp Psychiatry. 2017;47:48-60.

9. Hackett ML, Anderson CS, House A, et al: Interventions for treating depression after stroke.Cochrane Database Syst Rev. 2008:

10. Coupland C, Dhiman P, Morriss R, et al: Antidepressant use and risk of adverse outcomes in older people: population based cohort study. 2011;343:d4551.

11. Liu S, Yang ZH, Zhu XN, et al: Research Progress of TCM Therapies in Treating Post-Stroke Depression.Acta Chinese Medicine and Pharmacology. 2021;49:83-87.

12. Jiang DY, Ren PP, Li WH, et al: Study and Analysis of Hysteria.Jilin Journal of Chinese Medicine. 2020;40:880-884.

13. Shao Y: The effect of adding and subtracting Gan-Mai-Da-Zao decoction on postpartum women's sleep disorder and depressive state.Chinese Journal of Clinical Rational Drug Use. 2016;9:38-39.

14. Xia BM, Zhang HL, Xue WD, et al: Postpartum depression animal model in mice and effect of Yuejuganmaidazaotang on PPD model.Chinese Pharmacological Bulletin. 2015;000:1324$1327,1328$.

15. Xiang Y, Meng P, Zhang XL, et al: Research on Central Excitatory Pharmacological Effects of Ganmaidazao Decoction.Asia-Pacific Traditional Medicine. 2016;12:22-23.

16. Feng DD, Tang T, Lin XP, et al: Nine traditional Chinese herbal formulas for the treatment of depression: an ethnopharmacology, phytochemistry, and pharmacology review.Neuropsychiatr Dis Treat. 2016;12:2387-2402.

17. Chen HB, Zhou HG, Li.W.T., et al: Network pharmacology: A new perspective of mechanism research of traditional Chinese medicine formula.China Journal of Traditional Chinese Medicine and Pharmacy. 2019;34:2873-2876. 
18. Ru J, Li P, Wang J, et al: TCMSP: a database of systems pharmacology for drug discovery from herbal medicines.J Cheminform. 2014;6:

19. Shan Y, Feng X, Dong YF: The Research of Chemical Constituents and Pharmacological Activities of Triticum L. InThe Fourth National Symposium on Medicinal Botany and Phycology : October 2004; Nanjing China. 2004:376-384.

20. Zhang Y, Li Y, Wang J, et al: Advance in studies on effective components in wheat bran and their pharmacological activities.China Journal of Chinese Materia Medica. 2014;39(2) : 175-80.

21. Alam MA, Al-Jenoobi FI, Al-Mohizea AM, et al: Understanding and managing oral bioavailability: physiological concepts and patents. Recent Pat Anticancer Drug Discov. 2015;10:87-96.

22. Tian $\mathrm{S}$, Wang $\mathrm{J}$, Li Y, et al: The application of in silico drug-likeness predictions in pharmaceutical research.Adv Drug Deliv Rev. 2015;86:2-10.

23. Cui Q, Zhang YL, Ma YH, et al: A network pharmacology approach to investigate the mechanism of Shuxuening injection in the treatment of ischemic stroke.J Ethnopharmacol. 2020;257:

24. Fishilevich S, Zimmerman S, Kohn A, et al: Genic insights from integrated human proteomics in GeneCards.Database (Oxford). 2016;2016:baw030.

25. Szklarczyk D, Gable AL, Lyon D, et al: STRING v11: protein-protein association networks with increased coverage, supporting functional discovery in genome-wide experimental datasets.Nucleic Acids Res. 2019;47:D607-d613.

26. Tang Y, Li M, Wang J, et al: CytoNCA: a cytoscape plugin for centrality analysis and evaluation of protein interaction networks. 2015;127:67-72.

27. Kohl M, Wiese S, Warscheid B: Cytoscape: Software for Visualization and Analysis of Biological Networks.Methods in molecular biology (Clifton, NJ). 2011;696:291-303.

28. Hsin KY, Matsuoka Y, Asai Y, et al: systemsDock: a web server for network pharmacology-based prediction and analysis.Nucleic Acids Res. 2016;44:W507-513.

29. Seeliger $D$, de Groot BL: Ligand docking and binding site analysis with PyMOL and Autodock/Vina.J Comput Aided Mol Des. 2010;24:417-422.

30. Trott O, Olson AJ: AutoDock Vina: improving the speed and accuracy of docking with a new scoring function, efficient optimization, and multithreading.J Comput Chem. 2010;31:455-461.

31. Stevens W, Peneva D, Li JZ, et al: Estimating the future burden of cardiovascular disease and the value of lipid and blood pressure control therapies in China.BMC Health Serv Res. 2016;16:

32. Gong JY, Wu XQ, Mao JW, et al: Advanced in studies on antidepressant effect of flavonoids. Chinese Traditional and Herbal Drugs. 2011;042:195-200.

33. Samad N, Saleem A, Yasmin F, et al: Quercetin protects against stress-induced anxiety- and depression-like behavior and improves memory in male mice.Physiol Res. 2018;67:795-808.

34. Ma N, Li YJ, Fan JP: Research Progress on Pharmacological Action of Quercetin.Journal of Liaoning University of Traditional Chinese Medicine. 2018;20:221-224. 
35. Ishisaka M, Kakefuda K, Yamauchi M, et al: Luteolin shows an antidepressant-like effect via suppressing endoplasmic reticulum stress.Biol Pharm Bull. 2011;34:1481-1486.

36. Zhu S, Lei S, Zhou S, et al: Luteolin shows antidepressant-like effect by inhibiting and downregulating plasma membrane monoamine transporter (PMAT, Slc29a4).Journal of Functional Foods. 2019;54:440-448.

37. Burton MD, Rytych JL, Amin R, et al: Dietary Luteolin Reduces Proinflammatory Microglia in the Brain of Senescent Mice.Rejuvenation Res. 2016;19:286-292.

38. Liang YD, Tan YG, Zhang S, et al: Effect and mechanism of kaempferol on depression-like behavior in elderly rats with chronic stress depression. The Chinese Journal of Clinical Pharmacology. 2020;36;No.326:62-64.

39. Ben-Azu B, Nwoke EE, Aderibigbe AO, et al: Possible neuroprotective mechanisms of action involved in the neurobehavioral property of naringin in mice.Biomed Pharmacother. 2019;109:536-546.

40. Xu SL, Choi RC, Zhu KY, et al: Isorhamnetin, A Flavonol Aglycone from Ginkgo biloba L., Induces Neuronal Differentiation of Cultured PC12 Cells: Potentiating the Effect of Nerve Growth Factor.Evid Based Complement Alternat Med. 2012;2012:

41. Bortolato B, Hyphantis TN, Valpione S, et al: Depression in cancer: The many biobehavioral pathways driving tumor progression. Cancer Treat Rev. 2017;52:58-70.

42. Szelei A, Döme P: Cancer and depression: a concise review.Orv Hetil. 2020;161:908-916.

43. McFarland DC, Jutagir DR, Rosenfeld B, et al: Depression and inflammation among epidermal growth factor receptor (EGFR) mutant nonsmall cell lung cancer patients. 2019;28:1461-1469.

44. Iñiguez SD, Parise LF, Lobo MK, et al: Upregulation of hippocampal extracellular signal-regulated kinase (ERK)-2 induces antidepressant-like behavior in the rat forced swim test.Behav Neurosci. 2019;133:225-231.

45. Różycka A, Słopień R, Słopień A, et al: The MAOA, COMT, MTHFR and ESR1 gene polymorphisms are associated with the risk of depression in menopausal women. 2016;84:42-54.

46. Tan EC, Lim HW, Chua TE, et al: Investigation of variants in estrogen receptor genes and perinatal depression.Neuropsychiatr Dis Treat. 2018;14:919-925.

47. Liu HP: The effect of YinaoJieyu Prescription on the intervention of JAK2 and STAT3 proteins in the hippocampus of post-stroke depressed rats.Master thesis. Beijing University of Chinese Medicine, 2020.

48. Shinar D, Gross CR, Price TR, et al: Screening for depression in stroke patients: the reliability and validity of the Center for Epidemiologic Studies Depression Scale. 1986;17:241-245.

49. Zhang W, Zhang XA: A Novel Urinary Metabolite Signature for Non-invasive Post-stroke Depression Diagnosis.Cell Biochem Biophys. 2015;72:661-667.

50. Wang Y: Study on the antidepressant mechanism of acupuncture based on whole-transcriptome sequencing.PhD thesis. Beijing University of Chinese Medicine, 2017. 
51. Wang F, Zhang C, Fang YR: Ras signaling pathway and neural plasticity mechanism of depression.Journal of Shanghai Jiaotong University(Medical Science). 2015;35:

52. Wang JM, Yang Y, Liu XY, et al: Research Progress on Mechanism and Signaling Pathway Regulation of Chinese Materia Medica on Depression.Chinese Archives of Traditional Chinese Medicine. 2021;39:115-118.

\section{Figures}

Obtaining the active ingredients and targets of herbs from TCMSP
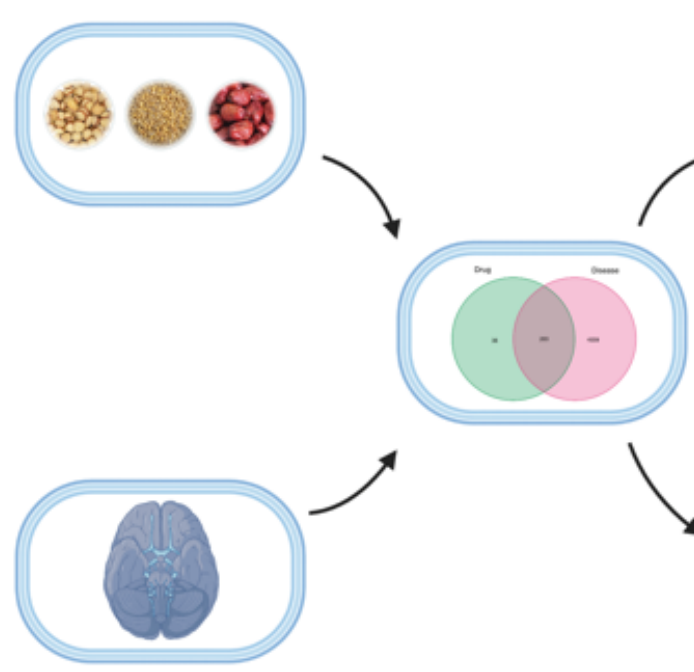

Access to disease-relevant targets from GeneCards and OMIM
Potential Gene Targets for TCM therapy PSD

Completing PPI networks and finding core targets with STRING and Cytoscape
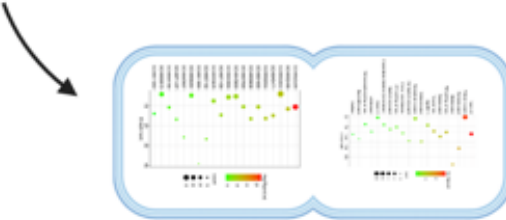
relationship graphs in Cytoscape

\section{Figure 1}

Detailed process of research design

Complete GO and KEGG pathway enrichment analysis using DAVID database
Building complex network

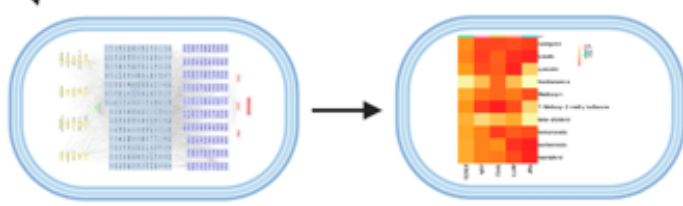

Docking results 
(a)

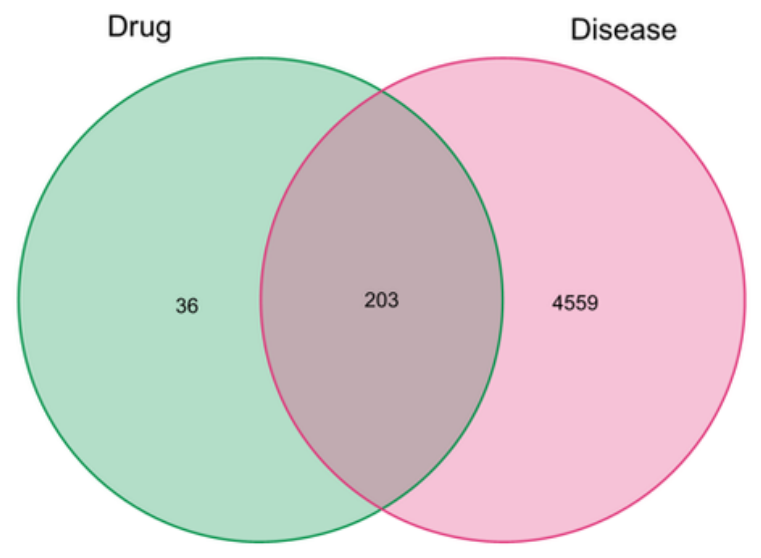

(c)

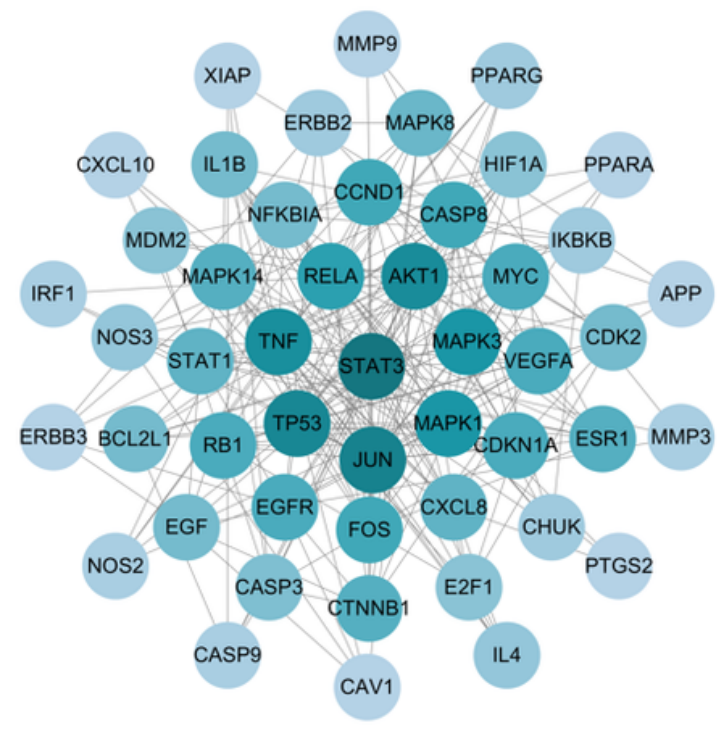

(b)

\begin{tabular}{|c|c|c|c|c|c|c|c|c|c|c|}
\hline CASPP & PLAU & Gsk38 & STAT1 & TP53 & Nos2 & OR1 & FASN & JUN & SLCEA3 & CDKNIA \\
\hline IL10RA & SLC2A4 & CYP19A1 & 1GF2 & ACACA & RASA 1 & PPARA & NOS3 & MTTP & $B a x$ & CAT \\
\hline SERPANE 1 & SREBF 1 & CYP1A1 & NR112 & PTGS1 & CASP7 & CAVI & RXRA & HSF1 & МАРКB & мир9 \\
\hline DRO2 & MMP1 & 1124 & CXCL10 & XIAP & IGFBP3 & RUNX2 & RAF1 & MMP10 & SELE & EGF \\
\hline AHR & ELK1 & PRKCA & MET & MAPK10 & II.2RA & ICAMI & NoO1 & VEGFA & E2F 1 & MAPK1 \\
\hline ІКВКВ & VCAM1 & PGR & $\mathrm{CCNA}_{2}$ & EGFR & PTGS2 & ESRI & COLIA1 & CCND1 & ESR2 & soo1 \\
\hline RB1 & COL3A1 & CYP3A4 & PRКCB & CHEK1 & PLAT & ERB83 & U18 & RELA & LTAAH & Mup3 \\
\hline HIF $1 \mathrm{~A}$ & MAPK14 & F7 & CHEK2 & GSR & CTSD & PPARG & MMP2 & IRF1 & $\mathrm{CxCL2}$ & BCLAL, \\
\hline CDAOLG & UGTIAT & NFE2L2 & AKRIC3 & APOB & CTNNB1 & IFNG & CXCL11 & CxcLs & HSD381 & CHUK \\
\hline CYPIA2 & BIRC5 & PTGES & KOR & CDK4 & CASP8 & MAPK3 & ERBB2 & MOM2 & $\mathrm{CCL}_{2}$ & FOS \\
\hline CASP3 & PARP1 & CDK2 & AKT1 & HSPB1 & GRIA2 & ILAA & GSTM1 & TNF & PPARD & $F_{3}$ \\
\hline 820 & PCNA & NR113 & spp1 & STAT3 & APP & ILEST & SULTIE: & ALOXS & CCNB1 & TOP1 \\
\hline $\mathrm{BCL} 2$ & NFKBAA & GJA1 & AR & Mrc & HMOX1 & LOLR & & & & \\
\hline
\end{tabular}

(d)

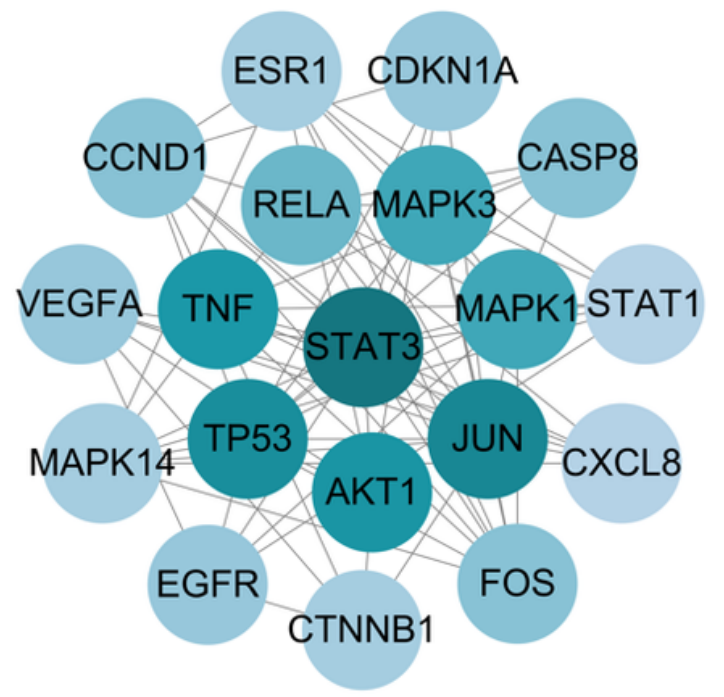

\section{Figure 2}

Drug-disease targets intersection Venn diagram and protein-protein interaction network. The darker the color means the larger the degree value, the more important it is in this network a) 203 intersection gene targets; b) a protein-protein network from STRING; c) one protein-protein cluster with 49 nodes and 230 edges; d) one protein-protein cluster with 19 nodes and 76 edges. 
(a)

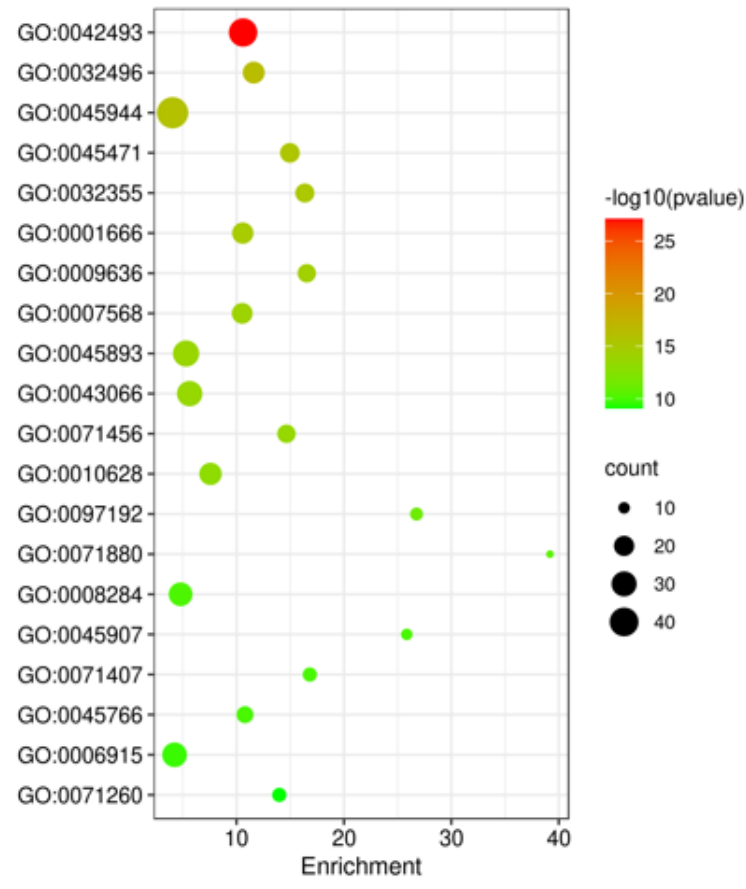

(c)

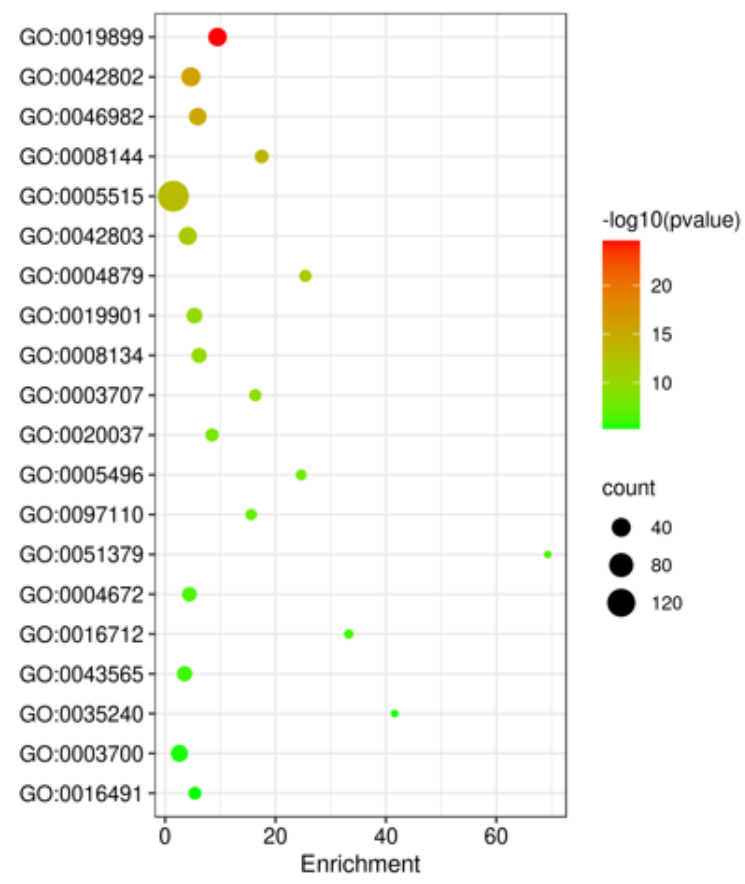

(b)

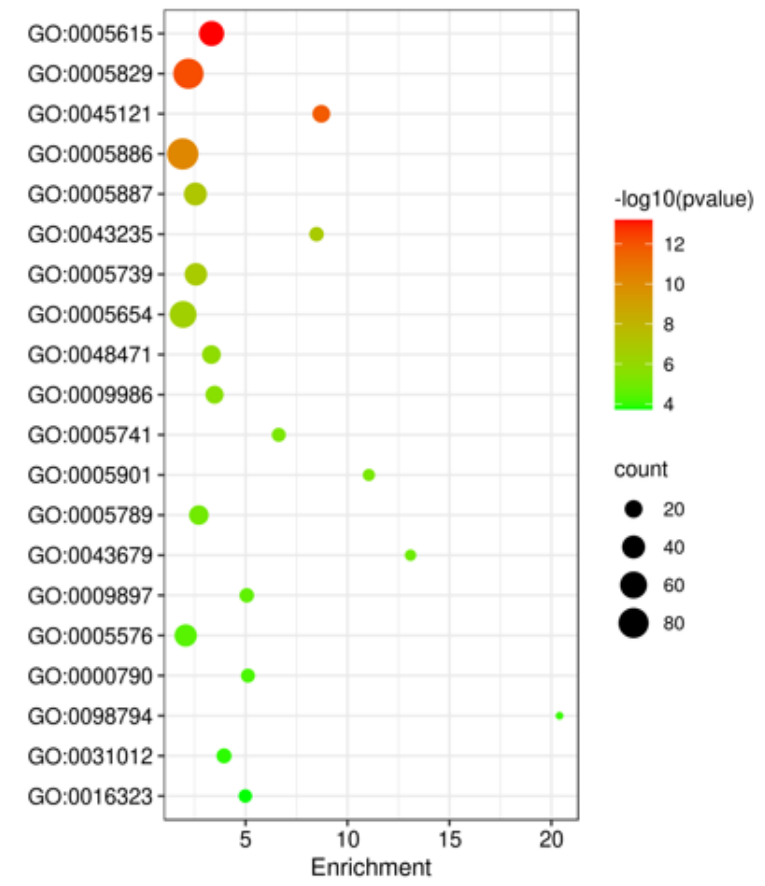

(d)

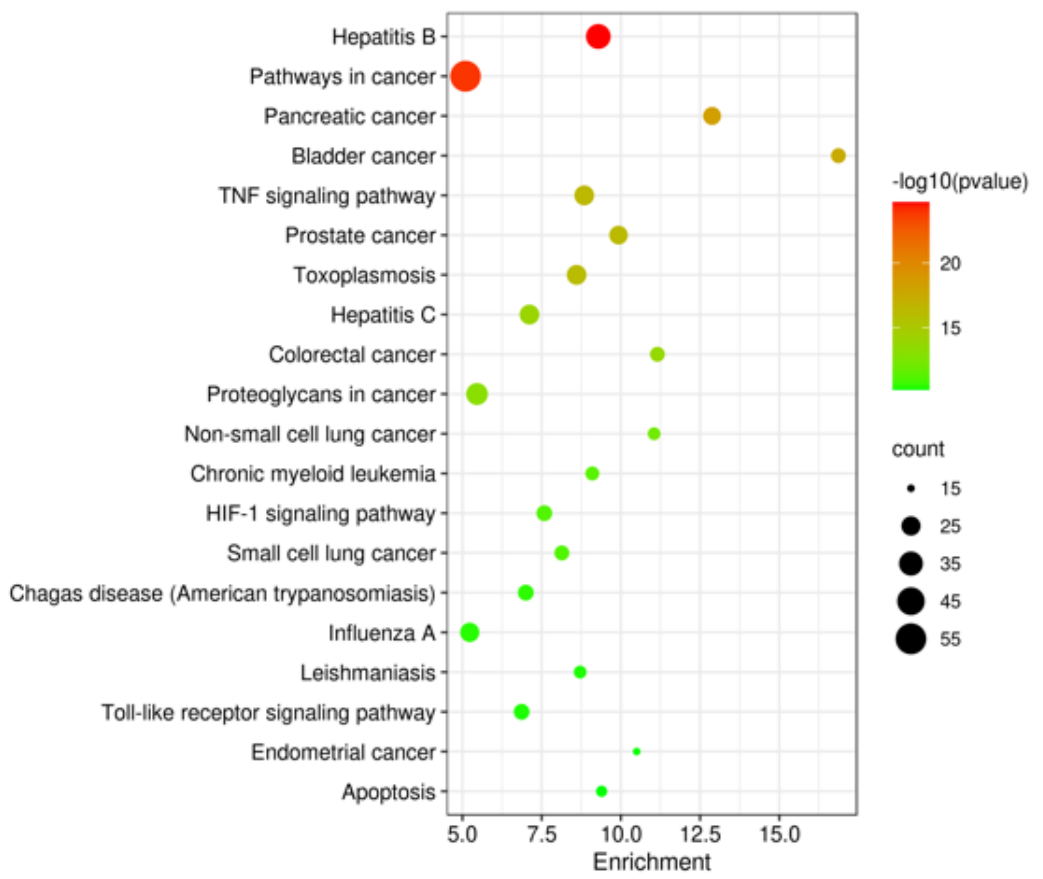

\section{Figure 3}

Bubble maps of GO and KEGG pathway enrichment analysis of Gan-Mai-Da-Zao decoction for PSD. a) GO: biological process; b) GO: cellular components; c) GO: molecular functions; d) KEGG pathway enrichment analysis 


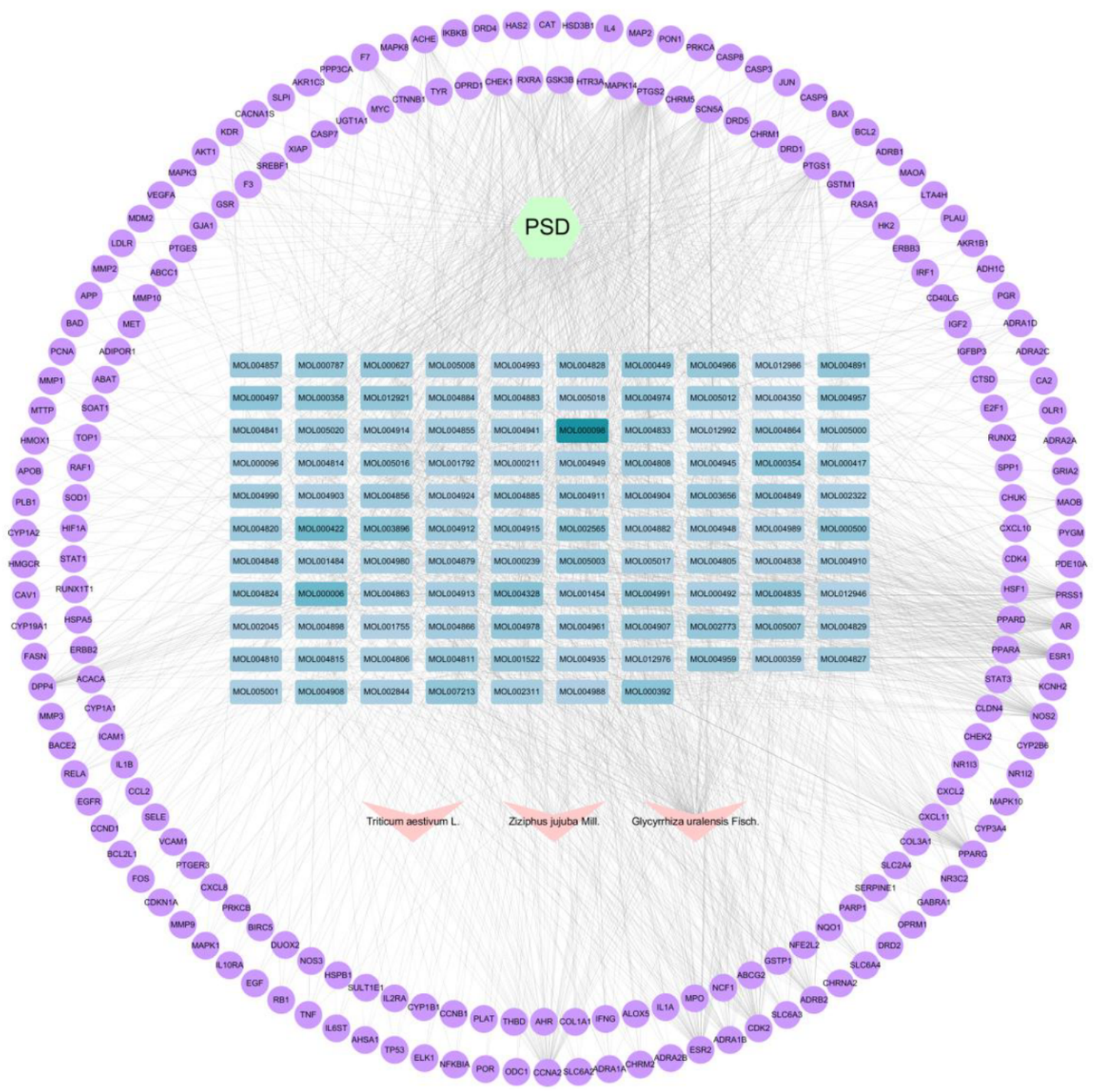

Figure 4

Disease-herb-compound-target (D-H-C-T) network of Gan-Mai-Da-Zao decoction against PSD. The purple circles represent genes, the green hexagons represent diseases, the pink V-shapes represent drugs, and finally, shades of blue rectangles represent active ingredients, the darker the color, the greater the degree value in the network, indicating greater importance. 
Zriphus jujuba Mail

\begin{tabular}{|c|c|c|}
\hline MOL005007 & MOL004808 & MOLo00449 \\
\hline MOLOCAOSO & MOL004604 & NoLoo4848 \\
\hline MOLOCES80 & MOL004961 & MOLoo4833 \\
\hline MOLOOAOB5 & MOLO04864 & MOL002311 \\
\hline MOL004824 & MOL004860 & MOL004806 \\
\hline MOLOC4993 & MOLO0 4855 & MOL005012 \\
\hline MOL000417 & MOL004907 & MOL005017 \\
\hline MOL001792 & MOLO0<968 & MOLOOU988 \\
\hline MOLOOCE27 & MOL004863 & NOLOOA828 \\
\hline MOL012946 & MOL002322 & NOLL00497 \\
\hline MOL004913 & MOL004691 & VOLO00211 \\
\hline
\end{tabular}

Gycyrnoza uralensis Fisch.

Tritcum aostivum L.

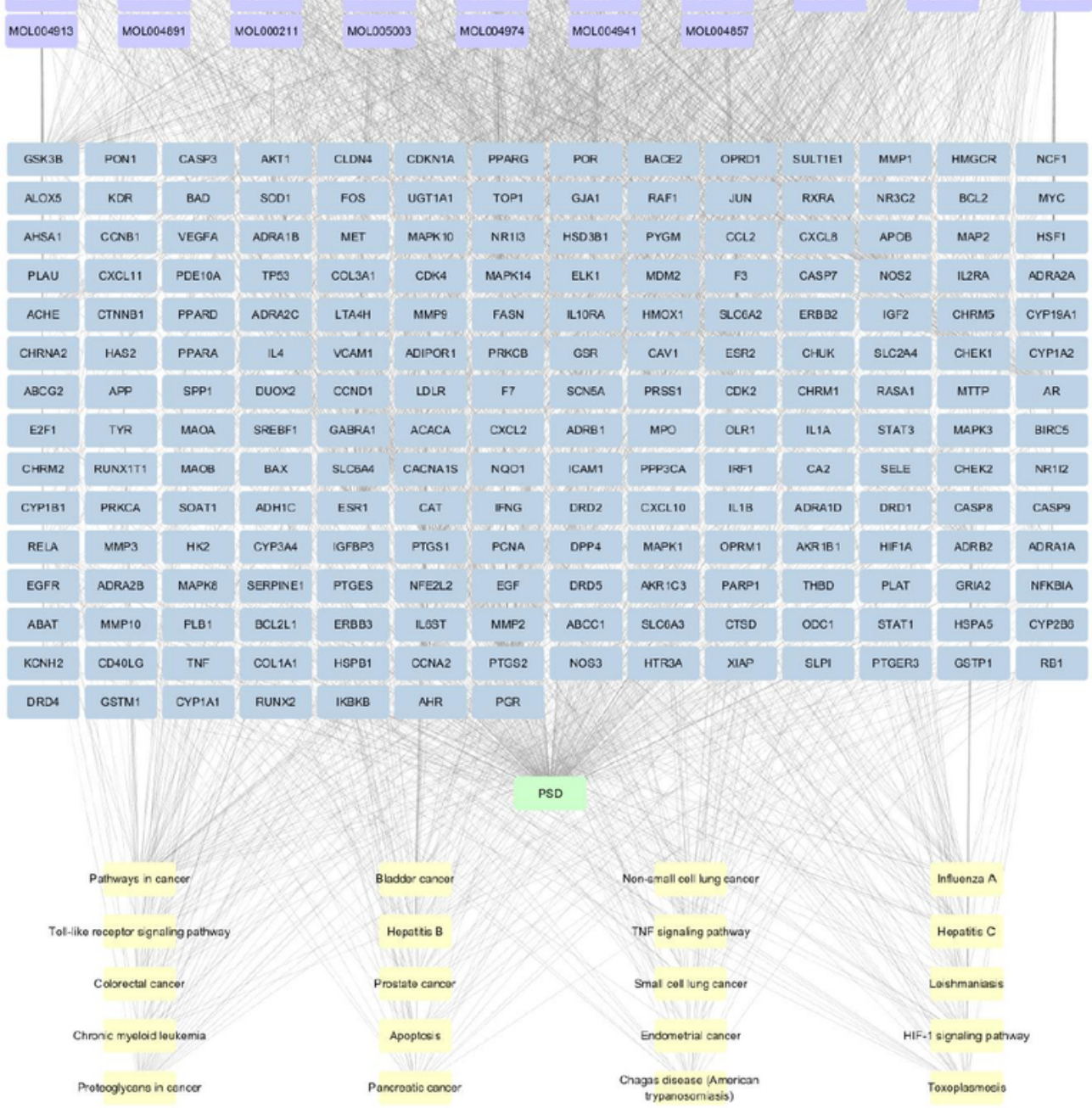

\section{Figure 5}

Network diagram containing pathway enrichment analysis. The different colored rectangles from top to bottom represent: herbal medicine, herbs, active ingredients, targets, disease, pathways 


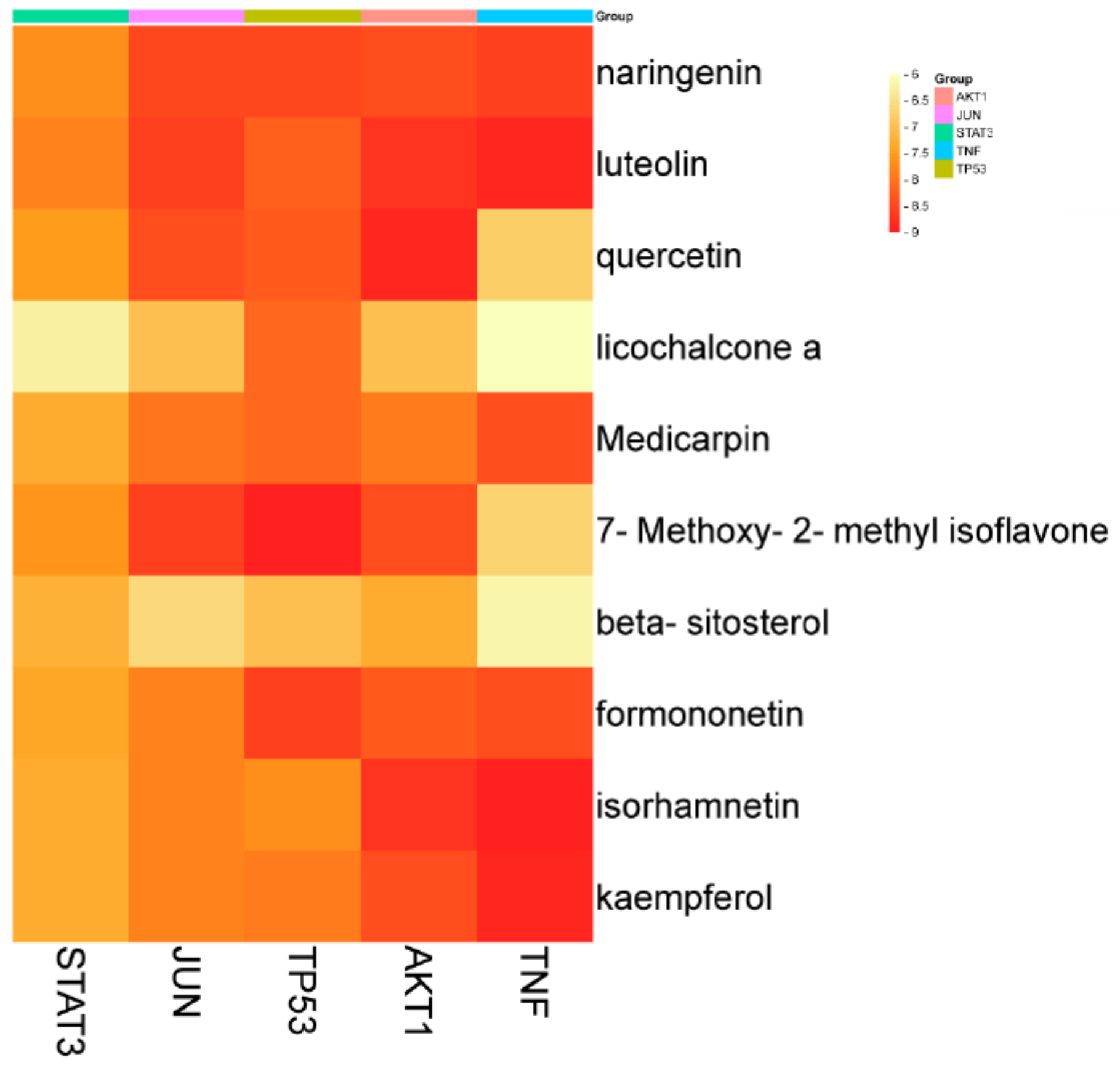

Figure 6

The heat map of the docking score 
(a)

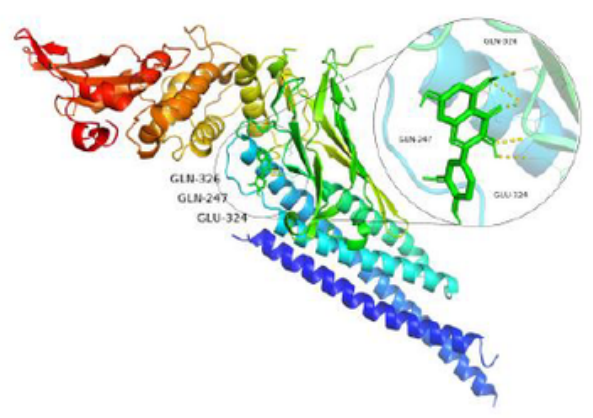

(c)

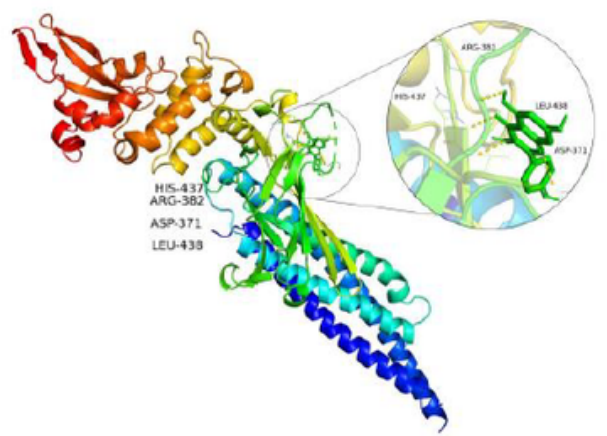

(e)

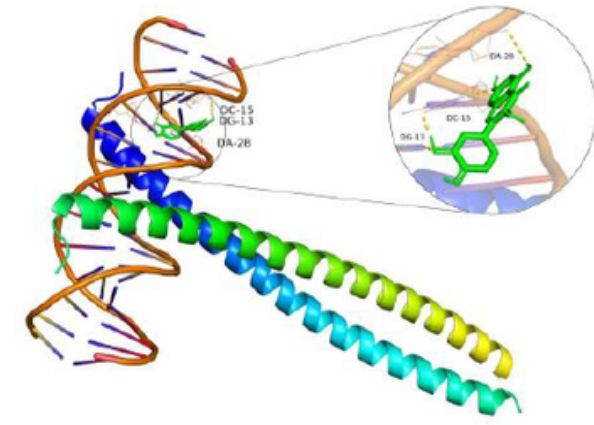

(g)

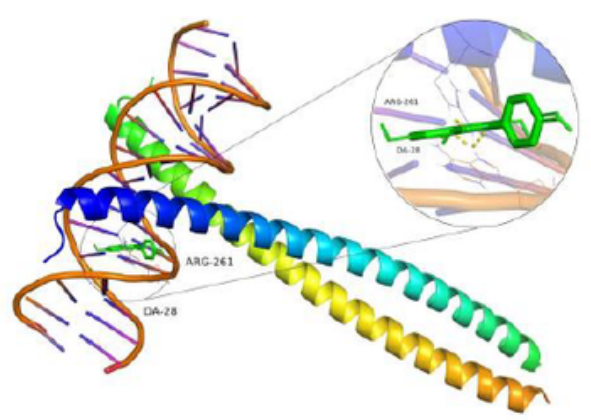

(b)

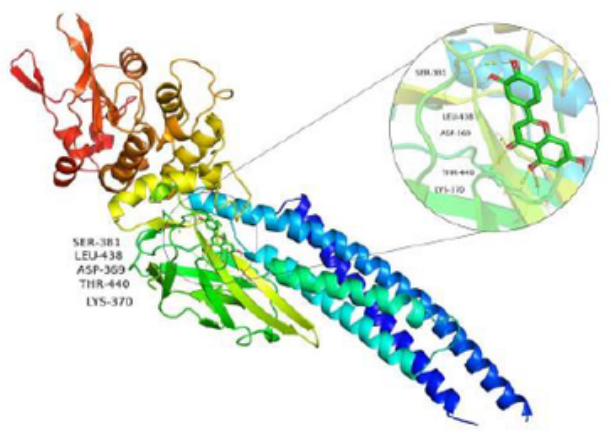

(d)

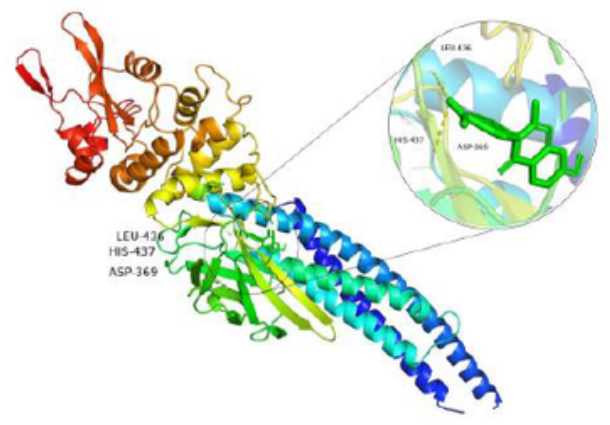

(f)

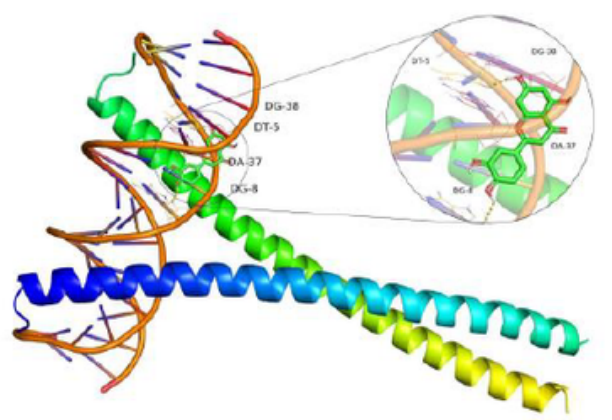

(h)

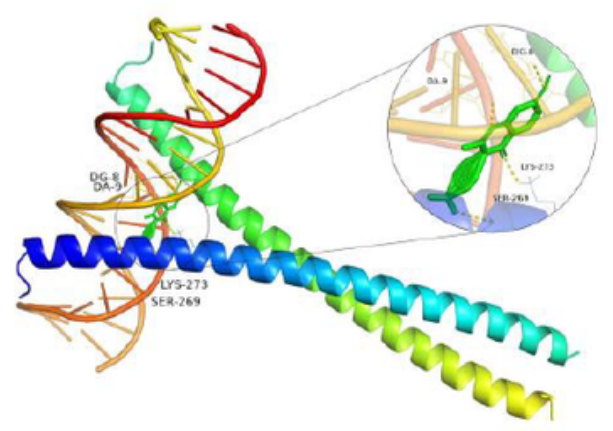

Figure 7

The docking complex consisting of two targets and four components. The colored irregular lines represent proteins, the green rod-like structures represent compounds, and each image shows the details of the docked parts. (a) STAT3-quercetin, (b) STAT3-luteolin, (c) STAT3-kaempferol, (d) STAT3-7-Methoxy2-methyl isoflavone, (e) JUN-quercetin, (f) JUN-luteolin, (g) JUN-kaempferol, (h) JUN-7-Methoxy-2-methyl isoflavone 


\section{Supplementary Files}

This is a list of supplementary files associated with this preprint. Click to download.

- SupplementaryMaterial.docx 